\title{
Modificación del abordaje quirúrgico en tumores estromales gástricos posterior a neoadyuvancia con Imatinib
}

\author{
Andrés Navarrete M. ${ }^{1,2,3}$, Dulce Momblán G. ${ }^{1}$, Ainitze Ibarzaval O. ${ }^{1}$, \\ Ricard Corcelles C. ${ }^{1}$, Marta Jiménez-Toscano ${ }^{1}$ y Antonio Lacy F. ${ }^{1}$
}

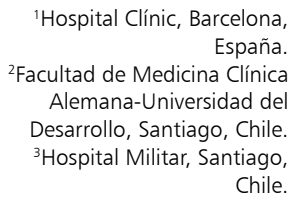

Recibido el 12 de abril de 2017 y aceptado para publicación el 4 de diciembre de

Correspondencia a:

Dr. Andrés Navarrete M. andres_navarr38@hotmail.com

\section{Change of surgical approach in gastrointestinal stromal tumors after} neoadyuvant therapy with Imatinib

Introduction: The treatment of high-risk gastrointestinal stromal tumors (GIST) is surgical. Results may change when using neoadjuvant. Objetive: To evaluated if the use of neoadjuvant therapy with imatinib can change the surgical approach in high risk gastrointestinal stromal tumors (GIST). Materials and Methods: A retrospective analysis was performed from a prospective collected database in Hospital Clinic of Barcelona between January 2002 and May 2016. Results: A total of 8 patients were analyzed with a mean age of $66.1 \pm 13.3$ years. The tumor location was upper third $37.5 \%$ (3) cases, $50 \%$ (4) in the middle third and $12.5 \%$ (1) in lower third. Because of high risk classification, location and the need of multivisceral resections, neoadjuvant therapy was indicated. The median time of neoadjuvant therapy was 30 weeks. In $87.5 \%$ (7) cases a change of surgical approach was achieved after the use of imatinib. In $100 \%$ of our series laparoscopic wedge resection was performed achieving negative margins of resection. The postoperative biopsy showed $51.2 \%$ of reduction of initial tumor size, resulting in statistical difference $(\mathrm{p}<0.01)$. All patients are alive and $100 \%$ of tumor related survival was achieved. Conclusion: Neoadjuvant therapy maybe can change the surgical approach of patients with high-intermediate risk gastric GIST by reducing tumor size. This response also eventually can achieve optimal oncological outcome.

Key words: GIST; gastric; neoadjuvant therapy; wedge resection.

\section{Resumen}

Introducción: El tratamiento de los tumores estromales gastrointestinales (GIST) de alto riesgo es quirúrgico. Su resultado podría variar al usarse neoadyuvancia. Objetivo: Evaluar si el uso de la terapia neoadyuvante con imatinib puede cambiar el abordaje quirúrgico en los tumores estromales gastrointestinales de alto riesgo. Materiales y Métodos: Se realizó un análisis retrospectivo en el Hospital Clínic de Barcelona entre enero de 2002 y mayo de 2016. Resultados: Se obtuvo un total de 8 pacientes. La edad media fue $66,1 \pm 13,3$ años. La ubicación del tumor fue de $37,5 \%$ (3) en el tercio superior, el $50 \%$ (4) en el tercio medio y el $12,5 \%$ (1) en el tercio inferior. Debido a la clasificación de riesgo alto, la ubicación y/o la necesidad de resecciones multiviscerales, se indicó, previa evaluación comité oncológico, realizar terapia neoadyuvante. La mediana de tiempo de neoadyuvancia fue de 30 semanas. En el 100\% (8) de los casos se logró un cambio de enfoque quirúrgico después de la utilización de imatinib. En todos los casos se realizó un resección local (7 laparoscópica y 1 endolaparoscópica) con márgenes negativos La biopsia posoperatoria mostró un promedio de $51,2 \%$ de reducción del tamaño tumoral inicial, lo que resultó en una diferencia estadística $(\mathrm{p}<0,01)$ con el tamaño inicial de las lesiones. Durante el seguimiento, tanto la sobrevida relacionada al tumor como la global, fue de un $100 \%$. Conclusión: La terapia neoadyuvante podría cambiar el abordaje quirúrgico de los pacientes con GIST gástrico de riesgo intermedio o alto mediante la reducción del tamaño tumoral, permitiendo realizar cirugías más económicas y logrando resultados oncológicos adecuados.

Palabras clave: GIST; gástrico; neoadyuvancia; resección local. 


\section{Introducción}

Los tumores estromales gastrointestinales representan el $80 \%$ de los tumores mesenquimáticos gastrointestinales y entre $0,1-3 \%$ de todos los tumores gastrointestinales ${ }^{1}$. La mejoría en los procedimientos endoscópicos principalmente con la aparición de la endosonografía y la utilización generalizada de técnicas de imagen de alta calidad, han aumentado las tasas de detección de este tipo de tumores. El estómago es el órgano más frecuentemente afectado, correspondiendo a un $60 \%-70 \%$ de los casos $^{2}$. Durante muchos años, la resección quirúrgica ha sido el pilar del tratamiento, siendo el único potencialmente curativo. Los GIST clasificados de bajo riesgo tienen excelentes resultados a largo plazo con un 90\%$100 \%$ de sobrevida a 5 años, pero el problema son aquellos de alto riesgo donde los resultados oncológicos son pobres. Aproximadamente, el 70\%-85\% de los GIST se determinan resecables al momento de presentación, dependiendo principalmente de la localización anatómica y/o el tamaño tumoral ${ }^{3-5}$. Sin embargo, en ausencia de estratificación del riesgo y sin uso de imatinib la resección quirúrgica sola tiene altas tasas de recurrencia; $50 \%$ a los 5 años y casi el $90 \%$ en el largo plazo ${ }^{6}$. Aproximadamente, el $10 \%-30 \%$ de los GIST diagnosticados tienen evidencia de invasión local y/o metástasis 7 . Un alto índice mitótico, gran tamaño, margen positivo de resección y la ruptura han sido identificados como factores de pronóstico negativo para la recurrencia y sobrevida ${ }^{3-5}$.

En los últimos años, una mejor comprensión del comportamiento biológico de los GIST seguido de la aprobación por la FDA del imatinib en 2002, han permitido una mejora en el manejo de los GIST y de este modo, una mejor sobrevida global ${ }^{8}$.

Estudios prospectivos relacionados al uso de imatinib como terapia neoadyuvante y adyuvante asociado a resección quirúrgica han mostrado resultados prometedores?.

El uso de imatinib ha demostrado alcanzar tasas de respuesta parcial o estabilidad de la enfermedad en casi un $80 \%$ de los pacientes con GIST localmente avanzado ${ }^{10-12}$. La terapia neoadyuvante con imatinib puede actuar en tres situaciones principales: preservación de órganos mediante la reducción del tamaño del tumor previo a la cirugía; cambiar un tumor irresecable inicialmente a un paciente operable $\mathrm{y}$, finalmente, como tratamiento de micrometástasis. El National Comprehensive Cancer Network Center (NCCN) y la Sociedad Europea de Oncología Médica recomiendan la terapia neoadyuvante con imatinib en pacientes en que la citorreducción preoperatoria sería beneficioso, incluyendo tumores marginalmente resecables o resecables con alto riesgo de resecciones multiviscerales ${ }^{13,14}$. Recientemente, se ha publicado que casi el $100 \%$ de los pacientes con ruptura tumoral durante la cirugía desarrollan metástasis abdominales durante el seguimiento ${ }^{15}$.

Por lo tanto, si el GIST es una lesión de gran tamaño y/o el riesgo de ruptura se considera alta, el tratamiento con imatinib debe iniciarse para aumentar las posibilidades de lograr resecciones R0 y disminuir el riesgo potencial de ruptura durante la cirugía.

El objetivo de nuestro estudio es evaluar si el uso de la terapia neoadyuvante con imatinib, puede cambiar el abordaje quirúrgico de los tumores de gran tamaño (alto riesgo) y también si la ruptura del tumor puede ser evitada.

\section{Materiales y Métodos}

Se realizó una revisión retrospectiva a partir de una base de datos recogida en forma prospectiva en el Hospital Clínic de Barcelona, entre enero de 2002 y mayo de 2016. De un total de 31 pacientes con GIST gástrico, 8 pacientes recibieron neoadyuvancia con imatinib y, posteriormente, fueron operados. Todos los pacientes tenían una endoscopía, endosonografía y una tomografía computarizada (TC) toracoabdominal como estudio preoperatorio. Se incluyeron todos los datos clínicos incluyendo datos demográficos y las características del tumor, el estudio histopatológico de pretratamiento, datos de morbimortalidad, muestra de biopsia operatoria y seguimiento. Los GIST se clasificaron mediante la clasificación de Fletcher ${ }^{20}$ antes y después de la terapia neoadyuvante, evaluando el tamaño del tumor y el índice mitótico. También se evaluaron los márgenes de resección en el $100 \%$ de casos. Todos los tumores se analizaron para la expresión de CD117 (c-Kit), CD34, actina de músculo liso (SMA) y S100. En todos los casos, la indicación del tratamiento neoadyuvante fue hecha por el comité oncológico. Tras el alta hospitalaria, todos los pacientes recibieron un seguimiento regular a 1 y 3 meses. Posteriormente, cada 3 meses durante los dos primeros años y luego cada 6 meses hasta completar 5 años. En los controles se solicitaron TC abdominal y endoscopía alternadamente cada 6 meses.

Imatinib fue el fármaco de elección en todos los pacientes con indicación de la terapia neoadyuvante, previo biopsia con c-kit $(+)$ de confirmación. La decisión para neoadyuvancia se basó en el tamaño de la lesión y/o el compromiso de órganos adyacentes 
con el objetivo de lograr una resección local y/o una cirugía con preservación de órganos. Antes del inicio de la terapia neoadyuvante se realizó una TC basal para evaluar no sólo el tamaño tumoral, sino también las características, como la densidad del tumor.

Después de iniciar la terapia neoadyuvante se midió la actividad antitumoral con TC en la semana 1 y luego a los 2 y 4 meses evaluando la reducción de tamaño y cambio en las características tumorales (Figura 1). La decisión de cirugía se basó en la obtención de dos TC sin cambios entre ellos. La respuesta a la terapia neoadyuvante se midió por la clasificación RECIST $1.1^{16}$.

Las variables categóricas se presentan como frecuencias (\%). Las variables continuas con distribuciones normales y no normales se presentan como media \pm desviación estándar y mediana (rango), respectivamente. Los parámetros se analizaron y se compararon mediante la prueba $\mathrm{t}$ de Student para datos independientes y para las variables continuas se establece el valor umbral de significación estadística de $\mathrm{p}<0,05$.

\section{Resultados}

Se analizaron un total de 8 pacientes ( 6 mujeres y 2 hombres). La edad media fue $66,1 \pm 13,3$ años (rango 42-82), con un IMC de 26,4 $\pm 3,9 \mathrm{~kg} / \mathrm{m}^{2}$. La mayoría de los pacientes fueron clasificados como ASA 2 (6/8) y sólo un paciente tenía cirugía previa. El síntoma de presentación fue dolor abdominal en el 62,5\% (5) de los casos, disfagia, hematemesis y pérdida de peso en $12,5 \%$ cada uno (Tabla 1). Todos los pacientes fueron estudiados con endoscopía y tomografía computarizada toracoabdominal. Además, la endosonografía con biopsia se realizó en el 100\% de los casos. La localización fue el tercio superior en $37,5 \%$ (3) de los casos, $50 \%$ (4) en el medio tercero y $12,5 \%$ (1) en el tercio inferior. El tamaño tumoral promedio fue $11,7 \pm 3,3 \mathrm{~cm}$ (rango de 8,0 a 16,0 $\mathrm{cm})$ y el índice mitótico fue $>5$ por $50 \mathrm{HPF}$ en $3 \mathrm{ca}-$ sos $\mathrm{y}<5$ por 50 HPF en los otros 5 casos. Todos los pacientes tenían c-KIT $(+)$. Con estas características, 7 casos fueron clasificados como tumores de alto riesgo y 1 caso como de riesgo intermedio. Después

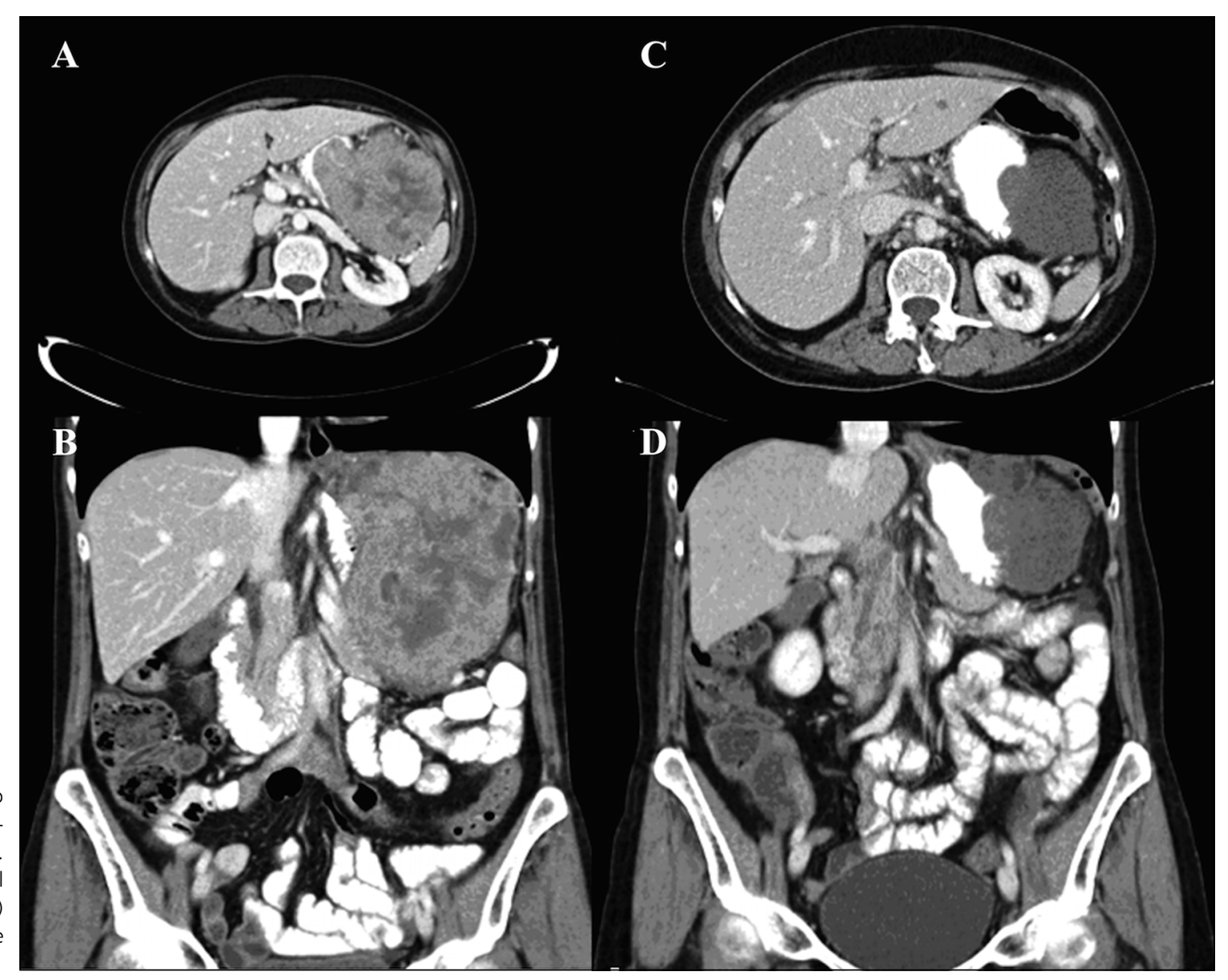

Figura 1. GIST gástrico de $15 \mathrm{~cm}$ previo a la terapia neoadyuvante (A, B). Reducción significativa del tamaño tumoral $(7 \mathrm{~cm})$ después de 6 meses de tratamiento (C, D). 
Tabla 1. Características generales

\begin{tabular}{|ccccccc|}
\hline & Edad & Sexo & IMC $\left(\mathbf{k g} / \mathbf{m}^{\mathbf{2}}\right)$ & ASA & Cirugías previas & Síntomas presentación \\
1 & 64 & M & 22,8 & 2 & No & Epigastralgia \\
2 & 54 & M & 21,2 & 1 & No & Hematemesis \\
3 & 74 & M & 32,0 & 3 & No & Epigastralgia \\
4 & 42 & H & 27,3 & 2 & No & Epigastralgia \\
5 & 80 & M & 23,2 & 2 & No & Epigastralgia \\
6 & 82 & M & 28,0 & 2 & No & Disfagia \\
7 & 67 & H & 31,1 & 2 & No & Pérdida de peso \\
\hline 8 & 66 & M & 26,1 & 2 & Sí & Dolor abdominal \\
\hline
\end{tabular}

IMC: Índice Masa Corporal. ASA: American Society of Anesthesiologists.

de evaluar la tomografía computarizada (tamaño y el compromiso de órganos vecinos) y las características histológicas del tumor; en los 8 casos se planteó como abordaje quirúrgico una gastrectomía total (3) o subtotal (5) para lograr una adecuada resección oncológica. Ningún paciente en nuestra serie fue clasificado inicialmente como irresecable, pero 4 de los pacientes necesitaban resecciones multiviscerales para lograr márgenes negativos. Por último, dado el alto riesgo de clasificación, localización y en 4 casos necesidad de resección multivisceral, se indicó la terapia neoadyuvante con imitanib. La duración media de la terapia con imatinib preoperatoria fue de 30 semanas (rango de 16-48 semanas) y no se observó progresión de la enfermedad en ningún caso (Figura 1a, 1b, 1c, 1d). Como se ha mencionado la terapia neoadyuvante se detuvo cuando dos TC no mostraron cambios entre ellas ( 7 casos) o porque el paciente presentó intolerancia a la medicación (1 caso a las 16 semanas).

Después de la terapia neoadyuvante todos los pacientes fueron sometidos a resección local laparoscópica. En 100\% (8) de los casos se logró un cambio de abordaje quirúrgico después del imatinib (Tabla 2).

Tabla 2. Características histológicas y modificaciones tanto de las características tumorales como del enfrentamiento o abordaje quirúrgico posterior al uso de imatinib

\begin{tabular}{|c|c|c|c|c|c|c|c|c|c|c|}
\hline \multirow{2}{*}{\multicolumn{2}{|c|}{$\begin{array}{c}\text { Tamaño } \\
\text { (cm) }\end{array}$}} & \multirow[b]{2}{*}{$\begin{array}{l}\text { Índice } \\
\text { mitótico }\end{array}$} & \multicolumn{3}{|c|}{ Pre-terapia neoadyuvante } & \multirow[b]{2}{*}{$\begin{array}{l}\text { \% Reducción } \\
\text { tamaño tumor }\end{array}$} & \multicolumn{4}{|c|}{ Posterapia neoadyuvante } \\
\hline & & & $\begin{array}{l}\text { Clasificación } \\
\text { de Fletcher }\end{array}$ & $\begin{array}{c}\text { Cirugía propuesta } \\
\text { inicialmente }\end{array}$ & $\begin{array}{c}\text { Duración } \\
\text { neoadyuvancia }\end{array}$ & & $\begin{array}{c}\text { Tamaño } \\
(\mathrm{cm})\end{array}$ & $\begin{array}{l}\text { Índice } \\
\text { mitótico }\end{array}$ & $\begin{array}{l}\text { Clasificación } \\
\text { de Fletcher }\end{array}$ & Cirugía \\
\hline 1 & 15,0 & $<5$ & Alto & $\begin{array}{l}\text { Gastrectomía subtotal + } \\
\text { colectomía parcial }\end{array}$ & 12 & 56,7 & 6,5 & $<5$ & Intermedio & $\begin{array}{l}\text { Resección } \\
\text { local }\end{array}$ \\
\hline 2 & 8,0 & $<5$ & Intermedio & Gastrectomía subtotal & 6 & 62,5 & 3,0 & $<5$ & Bajo & $\begin{array}{l}\text { Resección } \\
\text { local }\end{array}$ \\
\hline 3 & 10,0 & $<5$ & Alto & $\begin{array}{c}\text { Gastrectomía total }+ \\
\text { hepatectomía izquierda }\end{array}$ & 6 & 50 & 5,0 & $<5$ & Bajo & $\begin{array}{l}\text { Resección } \\
\text { local }\end{array}$ \\
\hline 4 & 12,0 & 20 & Alto & Gastrectomía subotal & 12 & 53,1 & 6,5 & 20 & Alto & $\begin{array}{l}\text { Resección } \\
\text { local }\end{array}$ \\
\hline 5 & 16,0 & $<5$ & Alto & $\begin{array}{l}\text { Gastrectomía total + } \\
\text { pancreatectomía distal }\end{array}$ & 6 & 47,5 & 7,6 & $<5$ & Intermedio & $\begin{array}{l}\text { Resección } \\
\text { local }\end{array}$ \\
\hline 6 & 7,0 & 20 & Alto & Gastrectomía total & 4 & 40 & 4,2 & $<5$ & Bajo & $\begin{array}{l}\text { Resección } \\
\text { endoscópica }\end{array}$ \\
\hline 7 & 11,0 & $<5$ & Alto & Gastrectomía subtotal & 6 & 45,6 & 5,0 & $<5$ & Bajo & $\begin{array}{l}\text { Resección } \\
\text { local }\end{array}$ \\
\hline 8 & 15,0 & 20 & Alto & $\begin{array}{l}\text { Gastrectomía total + } \\
\text { pancreatectomía distal } \\
\text { + esplenectomía }\end{array}$ & 8 & 73,3 & 11,0 & $<5$ & Alto & $\begin{array}{l}\text { Resección } \\
\text { local }\end{array}$ \\
\hline
\end{tabular}


Tabla 3. Resultados quirúrgicos y oncológicos

\begin{tabular}{|llccccccccc|}
\hline $\begin{array}{c}\text { Ruptura } \\
\text { tumoral }\end{array}$ & $\begin{array}{c}\text { Complicación } \\
\text { intraoperatoria }\end{array}$ & $\begin{array}{c}\text { Complicación } \\
\text { posoperatoria }\end{array}$ & Mortalidad & $\begin{array}{c}\text { Estancia } \\
\text { hospitalaria }\end{array}$ & $\begin{array}{c}\text { Terapia } \\
\text { adyuvante }\end{array}$ & Recurrencia & Metástasis & $\begin{array}{c}\text { Seguimiento } \\
\text { (meses) }\end{array}$ & Vivo \\
\hline 1 & No & No & No & No & 4 & Sí & No & No & 49 & Sí \\
2 & No & No & No & No & 3 & Sí & No & No & 24 & Sí \\
\hline 3 & No & No & No & No & 3 & Sí & No & No & 8 & Sí \\
\hline 4 & No & No & No & No & 5 & Sí & Sí & No & 24 & Sí \\
\hline 5 & No & No & No & No & 2 & Sí & No & No & 27 & Sí \\
\hline 6 & No & No & No & No & 5 & No & No & No & 12 & Sí \\
\hline 7 & No & No & No & No & 3 & Sí & No & No & 18 & Sí \\
\hline 8 & No & No & No & No & 3 & Sí & No & No & 2 & Sí \\
\hline
\end{tabular}

En todos los pacientes de nuestra serie se realizó una resección laparoscópica sin conversión. No tuvimos ningún caso de ruptura tumoral y en todos ellos se logró márgenes negativos de resección. En 1 de los pacientes la resección local fue endoscópica con apoyo laparoscópico ${ }^{17}$. No hubo complicaciones intraoperatorias (Tabla 3). La biopsia posoperatoria mostró una reducción tumoral de $11,7 \pm 3,3 \mathrm{~cm}$ a $6,1 \pm 2,8 \mathrm{~cm}$ logrando una media de reducción del 53,6\% (rango 40\%-73,3\%) del tamaño del tumor inicial, que resultó en una diferencia estadística significativa $(\mathrm{p}<0,01)$. Los 8 pacientes alcanzaron una respuesta parcial (RP) según criterios RECIST 1.1.

La clasificación de Fletcher también cambió después del tratamiento neoadyuvante; 4 de los 8 $(50 \%)$ pacientes clasificados inicialmente como alto o mediano riesgo pasaron a ser de bajo riesgo, 2 casos se mantuvieron de alto riesgo y los otros 2 casos cambiaron a ser tumores de riesgo intermedio (Tabla 2). La estancia hospitalaria fue de 3 días (rango 2-5), sin complicaciones posoperatorias ni mortalidad a 60 días. El uso de Imatinib como terapia adyuvante se introdujo en 7 de los 8 pacientes para completar 12 meses de tratamiento adyuvante. Se obtuvo un seguimiento medio de 20,5 meses (rango 2-48) (Tabla 3). Durante el seguimiento se observó 1 caso de recurrencia local después de terminar la terapia adyuvante (18 meses después de la cirugía). La paciente fue sometida a resección laparoscópica de la recurrencia y el imatinib se reinició durante 24 meses. No se observaron metástasis durante el seguimiento. En nuestra serie todos los pacientes están vivos y se logró el $100 \%$ de sobrevida relacionado con tumor.

\section{Discusión}

Los tumores estromales gastrointestinales son los tumores mesenquimáticos más frecuentes del tracto gastrointestinal. El único tratamiento potencialmente curativo es la resección quirúrgica. La cirugía puede obtener resultados oncológicos adecuados, especialmente en aquellas lesiones de tamaño pequeño y de bajo riesgo, que alcanzan una sobrevida a 5 años $>80 \%$. El problema principal son aquellos tumores de gran tamaño y de alto riesgo que podrían ser irresecables o necesitar resecciones multiviscerales para lograr márgenes negativos, con el riesgo no sólo de tener altas tasas de complicaciones posoperatorias, sino también un riesgo significativo de ruptura, resecciones incompletas y, por lo tanto, mayor tasas de recurrencia (50\% a los 5 años) $)^{6}$. Las lesiones de gran tamaño tienen más riesgo de ruptura, que es un punto clave para evitar la recurrencia. Los datos publicados muestran que, a largo plazo, el seguimiento de los pacientes sometidos solo a resección quirúrgica de GIST de alto riesgo, la cirugía no fue curativa en un gran número de pacientes con un 85$90 \%$ de resultados oncológicos adversos con mayor recurrencia, metástasis o muerte relacionada con tumor, logrando sobrevidas a 5 años menores que varían entre $32-78 \% \%^{17-19}$. Por lo tanto, mejorar el resultado de la resección es un punto clave en este tipo de pacientes. Afortunadamente, en los últimos 5 años, se ha hecho un progreso significativo en relación con la patogénesis molecular de los GIST que ha llevado a importantes avances en el conocimiento de esta enfermedad. De esta forma, se ha logrado mejorar los resultados oncológicos en los pacientes que anteriormente presentaban sobrevidas bajas. 
Uno de los principales pilares de estos avances se ha basado en los inhibidores de la tirosin kinasa principalmente para tumores irresecables, metastásicos y también para las lesiones de alto riesgo. Antes de iniciar el imatinib es fundamental contar con el estudio histológico para su confirmación, ya que los GIST tienen también diagnóstico diferencial.

En nuestra serie, se utilizó imatinib en los 8 casos con la intención de realizar resecciones locales, reducir la necesidad de resecciones multiviscerales, el riesgo de ruptura y para reducir la recurrencia a largo plazo. Imatinib como terapia neoadyuvante se utilizó en una media de 30 semanas (rango 16-48) similar a la reportada en los 10 centros de sarcoma EORTC STBSG que publicaron un tiempo medio de tratamiento preoperatorio de 40 semanas. Además mencionan que la respuesta óptima se obtiene incluso hasta los 12 meses, después de lo cual se observó una meseta ${ }^{20}$. Blanke et al., en un ensayo randomizado, investigó el resultado del tratamiento con imatinib en pacientes con GIST avanzado destacando que el tiempo medio de respuesta fue de 12 semanas. El estudio también mostró que el $75 \%$ de los pacientes respondieron al fármaco a las 23 semanas, con una duración media de la respuesta hasta 118 semanas. Estos hallazgos sugieren que el imatinib neoadyuvante debe administrarse durante al menos 3-6 meses ${ }^{21}$. Las tasas de respuesta parcial $(100 \%)$ que se logró en nuestra serie es alta al compararla con el $53,7 \%$ de las publicadas por Demetri et al. $(\mathrm{N}=120)$. Los mismos autores informan de un $50 \%$ a $96 \%$ de reducción del tamaño tumoral, similar al 53,6\% obtenido en nuestra serie $(11,7 \mathrm{~cm}$ a $6,1 \mathrm{~cm})^{11}$.

Se logró un $100 \%$ de cambio en el abordaje quirúrgico inicial. Los 4 casos de pacientes con un plan inicial de resecciones multiviscerales cambiaron a resecciones locales después de la terapia neoadyuvante. Los otros 4 pacientes también se sometieron a resecciones locales laparoscópicas sin ruptura intraoperatoria y sin complicaciones posoperatorias ni mortalidad. Después del tratamiento con imatinib se logró un $100 \%$ de resecciones locales laparoscópica, sin conversión a pesar de que el tamaño medio de los tumores era $>5 \mathrm{~cm}(5,8 \mathrm{~cm})$. El límite de tamaño superior para las resecciones GIST laparos-

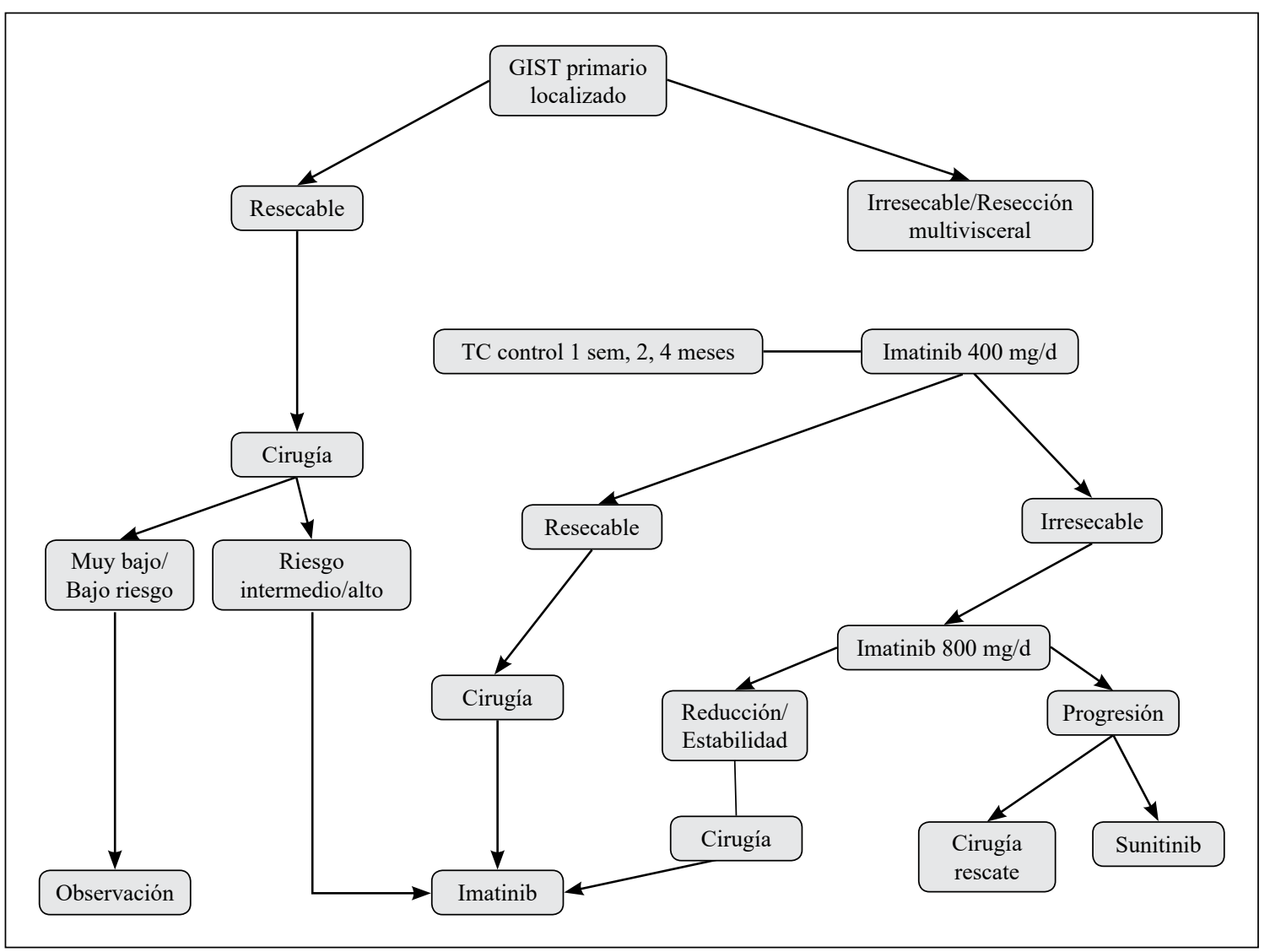

Figura 2. Algoritmo de manejo de GIST localizados. Se puede observar la indicación de imatinib en caso de lesiones irresecables o que necesiten resecciones multiviscerales (TC: Tomografía computada). 
cópicos ha sido continuamente modificado, llegando a $5 \mathrm{~cm}$ en la reciente actualización del National Comprehensive Cancer Network (NCCN) y en las directrices japonesas, pero el valor del abordaje laparoscópico para los GIST mayores de $5 \mathrm{~cm}$ permanece aún en evaluación, dada la falta de estudios a largo plazo sobre los resultados oncológicos ${ }^{2,11}$. La mayor parte de los casos recibieron terapia adyuvante durante 12 meses sobre la base de la clasificación preoperatoria. El único caso que no recibió imatinib posoperatorio fue debido a intolerancia clínica en el período neoadyuvante. Durante el seguimiento no se observó ninguna metástasis, pero tuvimos 1 caso de recurrencia local a pesar de que el paciente recibió 12 meses de la terapia adyuvante. La paciente fue sometida a resección laparoscópica de la lesión y reinició imatinib por otros 24 meses. Hay pocos datos publicados que evalúan los cambios en el enfoque quirúrgico o técnica en pacientes con GIST gástrico localmente avanzado que recibieron terapia neoadyuvante.

Las limitaciones de nuestro estudio son ser una muestra pequeña y el diseño retrospectivo. El seguimiento es de casi 2 años, pero un control a largo plazo sería ideal. A pesar de estas limitaciones, existen escasas publicaciones relativas al manejo de GIST gástrico de alto riesgo y el cambio potencial en el abordaje quirúrgico con el uso de la terapia con imatinib.

\section{Conclusiones}

El uso de inhibidores de tirosin kinasa como tera- pia neoadyuvante en GIST de riesgo intermedio-alto y/o lesiones de gran tamaño, podría reducir el tamaño tumoral lo que podría permitir realizar resecciones más económicas con preservación de órganos. Además, podría disminuir el riesgo de ruptura tumoral y complicaciones posoperatorias, logrando resultados oncológicos óptimos y modificando el grado tumoral. Es por esto que nos parece que el uso de neoadyuvancia con inhibidores de tirosin kinasa debiera estar dentro del manejo de estos tipos de GIST (Figura 2). El abordaje laparoscópico sería una opción viable y segura en este tipo de pacientes. Se necesitan ensayos clínicos para definir el manejo oncológico óptimo en este tipo de pacientes.

\section{Responsabilidades éticas}

Protección de personas y animales. Los autores declaran que para esta investigación no se han realizado experimentos en seres humanos ni en animales.

Confidencialidad de los datos. Los autores declaran que han seguido los protocolos de su centro de trabajo sobre la publicación de datos de pacientes.

Derecho a la privacidad y consentimiento informado. Los autores declaran que en este artículo no aparecen datos de pacientes.

\section{Conflicto de intereses}

Los autores declaran que no tienen conflictos de interés.

\section{Bibliografía}

1. Miettinen M, Sobin LH, Lasota J. Gastrointestinal stromal tumors of the stomach. A clinicopathologic, immunohistochemical, and molecular genetic study of 1765 cases with longterm follow-up. Am J Surg Pathol. 2005;29:52-68.

2. Demetri G, Von Mehren M, Antonescu C, DeMatteo R, Ganjoo K, Maki R, et al. NCCN Task Force report: update on the management of patients with gastrointestinal stromal tumors. J Natl Compr Canc Netw. 2010;8 Suppl 2:S1-41.

3. Fletcher CD, Berman JJ, Corless C, Gorstein F, Lasota J, Longley BJ, et al.
Diagnosis of gastrointestinal stromal tumors: a consensus approach. Hum Pathol. 2002;33:459-65.

4. Miettinen M, Lasota J. Gastrointestinal stromal tumors e definition, clinical, histological, immunohistochemical, and molecular genetic features and differential diagnosis. Virchows Arch. 2001;438:1-12.

5. Langer C, Gunawan B, Schuler P, Huber W, Fuzesi L, Becker H. Prognostic factors influencing surgical management and outcome of gastro- intestinal stromal tumours. Br J Surg. 2003;90:332-9.

6. DeMatteo RP, Lewis JJ, Leung D, Mudan SS, Woodruff JM, Brennan MF. Two hundred gastrointestinal stromal tumors: recurrence patterns and prognostic factors for survival. Ann Surg. 2000;231:51-8.

7. Miettinen M, Lasota J. Gastrointestinal stromal tumors: pathology and prognosis at different sites. Semin Diagn Pathol. 2006;23:70-83.

8. Pérez EA, Livingstone AS, Franceschi D, Rocha-Lima C, Lee DJ, Hodgson N, et al. Current incidence and outcomes of gastrointestinal mesenchymal tumors including gastrointestinal stromal tumors. J Am Coll. Surg. 2006;202:623-9.

9. Reynoso D, Trent JC. Neoadjuvant and adjuvant imatinib treatment in gastrointestinal stromal tumor: current status and recent developments. Curr Opin Oncol. 2010;22:330-5. 
ARTíCULO ORIGINAL

10. Joensuu H, Roberts PJ, Sarlomo-Rikala M, Andersson LC, Tervahartiala P, Tuveson D, et al. Effect of the tyrosine kinase inhibitor STI571 in a patient with a metastatic gastrointestinal stromal tumor. N Engl J Med. 2001;344:1052-6.

11. Demetri GD, von Mehren M, Blanke CD, Van den Abbeele AD, Eisenberg B, Roberts PJ, et al. Efficacy and safety of imatinib mesylate in advanced gastrointestinal stromal tumors. N Engl J Med. 2002;347:472-80.

12. van Oosterom AT, Judson I, Verweij J, Stroobants S, Donato di Paola E, Dimitrijevic S, et al. Safety and efficacy of imatinib (STI571) in metastatic gastrointestinal stromal tumours: a phase I study. Lancet 2001;358(9291):1421-3.

13. Demetri GD, Benjamin RS, Blanke CD, Blay JY, Casali P, Choi H, et al. NCCN Task Force report: management of patients with gastrointestinal stromal tumor (GIST): update of the NCCN clinical practice guidelines. J Natl Compr Cancer Netw. 2007;5 Suppl 2:S1-29.

14. Cassier PA, Dufresne A, Arifi S, El Sayadi H, Labidi I, Ray-Coquard I, et al. Imatinib mesilate for the treatment of gastrointestinal stromal tumour. Expert Opin Pharmacother. 2008;9:1211-22.

15. Hohenberger $P$, Ronellenfitsch $U$, Oladeji O, Pink D, Ströbel P, Wardelmann E, et al. Pattern of recurrence in patients with ruptured primary gastrointestinal stromal tumour. Br J Surg. 2010;97:1854-9.

16. Bogaerts JI, Ford R, Sargent D, Schwartz LH, Rubinstein L, Lacombe D, et al. Individual patient data analysis to assess modifications to the RECIST criteria. Eur J Cancer 2009;45:248-60.

17. Navarrete A, Momblan D, FernándezEsparrach G, Delgado S, Jiménez M, et al. Endoscopic full-thickness resection of esophagogastric junction gastrointestinal stromal tumor assisted by laparoscopy after neoadjuvant therapy. Endoscopy 2016;48(S01): E112-4.
18. Roberts PJ, Eisenberg B. Clinical presentation of gastrointestinal stromal tumors and treatment of operable disease. Eur J Cancer 2002;38(Suppl 5):S37-8.

19. Heinrich MC, Blanke CD, Druker BJ, Corless CL. Inhibition of KIT tyrosine kinase activity: a novel molecular approach to the treatment of KIT-positive malignancies. J Clin Oncol. 2002;20: 1692-703.

20. Rutkowski P, Gronchi A, Hohenberger P, Bonvalot S, Schöffski P, Bauer S, et al. Neoadjuvant imatinib in locally advanced gastrointestinal stromal tumors (GIST): the EORTC STBSG experience. Ann Surg Oncol. 2013;20:2937-43.

21. Blanke CD, Joensuu H, Demetri GD, Heinrich MC, Eisenberg B, Fletcher J, et al. Outcome of advanced gastrointestinal stromal tumor (GIST) patients treated with imatinib mesylate: four- year followup of a phase II randomized trial. ASCO Gastrointestinal Cancers Symposium Proceeding 2006;(abstr 7). 